Aim of the study: Epstein-Barr virus-related post-transplant lymphoproliferative disorder (EBV-PTLD) is a serious complication after stem cell transplantation (SCT) and the number of patients at risk is increasing over time. Available data do not reflect general practice of diagnosis and treatment of this complication.

Material and methods: In 2009 a survey on management of the pre-emptive strategy of EBV infection was done and results from 74 European transplant centers were registered and analyzed. Results: Regular monitoring for EBV after SCT is done by most of the participating centers (73\%). In $68 \%$ of them the monitoring is performed in all alloSCT patients, while in remaining centers it is done in high-risk patients only. Quantitative EBV-DNA is performed in $97 \%$ of centers, mainly in whole blood (78\%) and usually repeated once a week (60.9\%). The monitoring for EBV reactivation is performed for a period of 3 months (37\%) to 6 months (30\%) or adjusted to risk factors (20\%). Rituximab as a pre-emptive therapy for EBV-PTLD is routinely administered in $80 \%$ of responding centers. The number of EBVDNA copies as an indicator for preemptive therapy with rituximab varies between the centers.

Conclusions: The strategy of management of EBV infection exists in most of the responding centers. Different approaches regarding indications for preemptive therapy are seen between centers: rituximab is administered as preemptive therapy in most participating transplant centers.

Key words: Epstein-Barr virus, posttransplant lymphoproliferative disease, hematopoietic stem cell transplantation, pre-emptive strategy.

\section{Strategy of pre-emptive management of Epstein-Barr virus post-transplant lymphoproliferative disorder after stem cell transplantation: results of European transplant centers survey}

\author{
Lidia Gil', Jan Styczyński², Mieczysław Komarnicki ${ }^{1}$
}

1Department of Hematology, Poznan University of Medical Sciences, Poznan, Poland 2Department of Pediatric Hematology and Oncology, Collegium Medicum, Nicolaus Copernicus University, Bydgoszcz, Poland

\section{Introduction}

Epstein-Barr virus-related post-transplant lymphoproliferative disorder (EBV-PTLD) is a serious complication after stem cell transplantation (SCT), associated with as high as $90 \%$ rate of mortality only a decade ago [1]. The varying incidence ranging from $0.45 \%$ to $29 \%$ of reported EBV-PTLD in SCT recipients is related mainly to the degree of graft manipulation and intensity of immunosuppression used, which may cause uncontrolled proliferation of B lymphocytes driven by EBV $[2,3]$. Identified risk factors for development of EBV-PTLD include SCT from unrelated, mismatched or haploidentical donors, cord blood SCT, T-depleted SCT (in vivo or in vitro), use of antithymocyte globulin (ATG), splenectomy or serological EBV mismatch between donor and recipient $[1,4]$. Among high-risk patients the incidence of PTLD can reach $29 \%$ vs. $0.45 \%$ for patients transplanted from identical siblings. EBV-related PTLD may occur early during the first year after $\mathrm{SCT}$, with the highest incidence within the third month, or late, even many years after transplantation. Disease is highly heterogeneous: from indolent self-limited form to disseminated lymphoproliferation. Early diagnosis and treatment is crucial to overcome poor prognosis of this condition. According to the current definition proven EBV disease (PTLD or other end organ disease) requires EBV detection from an affected organ by biopsy or other appropriate invasive procedure together with symptoms or signs from this organ [2, 3]. Probable EBV disease is defined as significant lymphadenopathy (or other end-organ disease) with high EBV load in the blood, in the absence of other etiologic factors or established disease. EBV-DNA-emia is related to detection of EBV-DNA in the blood [3]. The correlation between the number of EBV-DNA copies and PTLD development has been described and regular monitoring of the EBV load allows one to start early, preemptive treatment, despite no consensus regarding the threshold value for such intervention. Current recommendations suggest use of the monoclonal antiCD20 antibody rituximab for preemptive treatment $[3,5]$.

Today with increased use of transplants from alternative donors, the population at risk of EBV-PTLD is increasing over time. Available data are largely based on series of cases or single center observations and do not reflect general practice of diagnosis and treatment of this complication [2]. The present study was designed to assess the current strategy of diagnosis and preemptive use of rituximab for EBV-PTLD in European transplant centers (TC) with respect to published guidelines [3]. 


\section{Material and methods}

In order to obtain information about the diagnostic strategy and preemptive treatment practices for EBV-PTLD in Europe, a questionnaire was prepared and sent to European transplant centers. The questionnaire consisted of several questions concerning indications for EBV monitoring, method and rate of monitoring, and indications for preemptive treatment with rituximab. Data from participating transplant centers registered on specific forms were analyzed.

\section{Results}

Seventy-four transplant centers, including 7 Polish centers, responded to the query. It constituted about $12 \%$ of all questioned TC, which is higher than average. Responses from 10 centers were excluded due to lack of any anti-EBV specific strategy. Thus, further analysis was done based on data from 64 centers.

\section{EBV monitoring}

Regular monitoring for EBV after SCT is done by the majority of responding centers $47 / 64$ (73\%), but in 17/64 (27\%) is restricted to clinical suspicion of developing EBV-PTLD. A half of centers (50\%) perform monitoring in all alloSCT patients; the rest do so only in high-risk patients (Table 1). Single centers consider EBV monitoring in patients after autologous SCT, which seems to be essential especially for autoimmune disorders or patients treated with purine analogues or alemtuzumab before transplant.

\section{Technique}

According to current recommendations, EBV-DNA assay is based on quantitative polymerase chain reaction (PCR), and this method is used in most of the responding transplant centers (62/64, i.e. 97\%), while qualitative PCR is used in the other 2 centers.

\section{Source}

At present, there are no data concerning the best biological source to be used. However, real time PCR of the EBV-DNA in whole blood is preferred by the majority of TC (50/64 i.e. $78 \%$ ), and is rarely done in plasma (9/64, i.e. $14 \%$ ), blood lymphocytes (4/64, i.e. $6 \%$ ) or serum (1 center).

\section{Frequency of monitoring}

The test is repeated twice a week in 8/64 TC (12.5\%), once a week in 39/64 (60.9\%), once per 2 weeks in 8/64 (12.5\%), once a month in 5/64 (7.8\%) and adjusted to the presence of risk factors in 4/64 (6.2\%) TC. Weekly monitoring of EBVDNA-emia, recommended for patients at high risk of PTLD, allows early detection of high EBV load and follow-up of viral load kinetics necessary for therapeutic decisions [3].

The monitoring for EBV reactivation after $\mathrm{SCT}$ is performed for a period of less than 3 months in 2/64 (3\%) TC, 3 months in 22/64 (34\%), 6 months in 19/64 (30\%), 12 months in 8/64 $(12 \%)$ and adjusted to risk in $13 / 64$ (20\%). Since the highest incidence of EBV-PTLD is seen within the first three months after transplant, longer monitoring of EBV-DNA should be considered in patients with graft-versus-host dis- ease, after haploidentical SCT and with a history of EBV reactivation.

\section{Preemptive treatment}

Preemptive treatment is defined as any therapy given to a patient with EBV-DNA-emia. Treatment of EBV disease is defined as any therapy applied to a patient with proven or probable EBV disease [3].

The monoclonal anti-CD20 antibody rituximab is currently the most widely used preemptive therapy for post-transplant EBV infection. Available data indicate that rituximab reduces PTLD development and risk of death due to EBV-PTLD [2]. The drug as a pre-emptive therapy for EBV-PTLD is routinely administered in 51/64 (80\%) of participating European transplant centers.

The threshold value of EBV-DNA copies as an indicator for pre-emptive therapy with rituximab was given by $60 \mathrm{TC}$, but varied between the centers (Table 2). 19/60 (31.6\%) TC declared that they made the decision of preemptive therapy based on symptoms and signs together with EBV-DNA results or increasing values of EBV-DNA-emia.

\section{Discussion}

The study shows that many European transplant centers realize the risk of development of EBV-related PTLD. TC recognize risk factors for this disease and perform regular screening and monitoring of EBV-DNA. In $80 \%$ of responding centers, rituximab is used as preemptive therapy for EBV-DNAemia. Our analysis indicates a change in approach to EBV-relat-

Table 1. Indication for monitoring of EBV-DNA according to type of transplant

\begin{tabular}{|l|l|}
\hline Type of transplant & Number of centers (\%) \\
\hline all alloSCT & $32 / 64(50.0 \%)$ \\
\hline MUD-SCT & $49 / 64(76.5 \%)$ \\
\hline T-depletion in vitro & $40 / 64(62.5 \%)$ \\
\hline T-depletion in vivo & $53 / 64(82.8 \%)$ \\
\hline family mismatched SCT & $46 / 64(71.9 \%)$ \\
\hline cord blood SCT & $48 / 64(75.0 \%)$ \\
\hline other & $3($ SAA, EBV mismatch, \\
\hline
\end{tabular}

alloSCT - allogeneic stem cell transplantation; MUD - matched unrelated donor; SAA - severe aplastic anemia; EBV - Epstein-Barr virus

Table 2. Threshold value of EBV-DNA copies as an indicator for preemptive treatment with rituximab

\begin{tabular}{|ll|}
\hline Number of EBV-DNA copies/ml & Number of centers \\
\hline $1 \times 10^{3}$ & $19(31.7 \%)$ \\
\hline $5 \times 10^{3}$ & $1(1.7 \%)$ \\
\hline $1 \times 10^{4}$ & $20(33.3 \%)$ \\
\hline $4-5 \times 10^{4}$ & $6(10 \%)$ \\
\hline $1 \times 10^{5}$ & $9(15 \%)$ \\
\hline other (risk factors, increasing value) & $5(8.3 \%)$ \\
\hline
\end{tabular}

EBV-DNA - Epstein-Barr virus deoxyribonucleic acid 
ed complications in stem cell transplant patients. Only a decade ago, before the current methods of anti-EBV and PTLD therapy including use of rituximab were introduced, the mortality from overt EBV-PTLD after SCT was higher than 80\% $[6,7]$. Current recommended dosing of rituximab in preemptive therapy is $375 \mathrm{mg} / \mathrm{m}^{2}$ of body surface area, 1-2 doses [3].

At present, there is no consensus when to start preemptive treatment regarding the threshold value of EBV-DNA. The value of EBV-DNA-emia alone as an indicator for such therapy is debatable, since a spontaneous decrease in the number of EBV copies was observed [6]. Some responders from participating TC suggest analysis of EBV-DNA-emia kinetics for about 2 weeks and preemptive treatment when persistent or increasing EBV-DNA-emia value is observed.

With respect to the threshold value being the indication for preemptive use of rituximab, about $1 / 3$ of centers use the value of $10^{3}$ copies $/ \mathrm{ml}$, and about $1 / 3$ use $10^{4}$ EBV-DNA copies/ml. Based on this study and previous experience [6], a higher value than $10^{3}$ EBV-DNA copies/ml or, at least, a repetitive number of EBV-DNA copies, should be recommended for start of rituximab preemptive therapy.

Rituximab seems to be the most important approach in preemptive therapy of EBV-DNA-emia due to large availability to virtually all transplant centers, safety and ease of administration and high rate of good overall response, as reported in our previous report [2]. Rituximab is known to be a safe and well-tolerated drug. Effectiveness of rituximab in preemptive intervention of EBV-DNA-emia is higher than its use in therapy of probable or proven EBV-PTLD [2]. The treatment should be managed however with caution, because of profound B-cell depletion and delay in Blymphocyte recovery and function [8]. Other methods of preemptive strategy include use of EBV-specific cytotoxic T lymphocytes, mostly as a second line treatment, or reduction of immunosuppression, which is not possible in all cases $[3,5,9,10]$.

Another important finding of this study is the almost universal approach to diagnosis of EBV, based on quantitative PCR assay. It seems that this assay is available in European countries as a "gold standard" practice. Targeted EBV monitoring of high-risk patients is a useful strategy to control EBVrelated PTLD [6]. A new method being developed and currently studied in diagnostics in solid organ recipients at risk is gene expression analysis using microarrays [11], but this method is nowadays unavailable as a common practice for all centers.

\section{Conclusions and future direction}

An EBV-PTLD strategy exists in most of the responding centers. Differences in the approach regarding indications for preemptive therapy are seen between centers; however, rituximab is administered as preemptive therapy in $80 \%$ of participating transplant centers.

The most important issue to be solved is the indication and method of preemptive therapy and a prospective study is needed to answer this question. As a continuation of the current survey, a retrospective study on the management of EBV-PTLD treated with rituximab after stem cell transplantation is under preparation.

\section{References}

1. Curtis RE, Travis LB, Rowlings PA, et al. Risk of lymphoproliferative disorders after bone marrow transplantation: a multi-institutional study. Blood 1999; 94: 2208-16.

2. Styczynski J, Einsele H, Gil L, Ljungman P. Outcome of treatment of Epstein-Barr virus-related post-transplant lymphoproliferative disorder in hematopoietic stem cell recipients: a comprehensive review of reported cases. Transpl Infect Dis 2009; 11: 383-92.

3. Styczynski J, Reusser P, Einsele H, de la Camara R, Cordonnier C, Ward KN, Ljungman P, Engelhard D. Management of HSV, VZV and EBV infections in patients with hematological malignancies and after SCT: guidelines from the Second European Conference on Infections in Leukemia. Bone Marrow Transplant 2009; 43: 757-70.

4. Sundin M, Le Blanc K, Ringden O, Barkholt L, Omazic B, Lergin C, Levitsky $\mathrm{V}$, Remberger $\mathrm{M}$. The role of HLA mismatch, splenectomy and recipient Epstein-Barr virus seronegativity as risk factors in post-transplant lymphoproliferative disorder following allogeneic hematopoietic stem cell transplantation. Haematologica 2006; 91: 1059-67.

5. Tomblyn M, Chiller T, Einsele H, et al. Guidelines for preventing infectious complications among hematopoietic cell transplantation recipients: a global perspective. Biol Blood Marrow Transplant 2009; 15: 1143-238.

6. Omar H, Hagglund H, Gustafsson-Jernberg A, et al. Targeted monitoring of patients at high risk of post-transplant lymphoproliferative disease by quantitative Epstein-Barr virus polymerase chain reaction. Transpl Infect Dis 2009; 11: 393-9.

7. Landgren O, Gilbert ES, Rizzo JD, et al. Risk factors for lymphoproliferative disorders after allogeneic hematopoietic cell transplantation. Blood 2009; 113: 4992-5001.

8. Masjosthusmann K, Ehlert K, Eing BR, Roth J, Koehler G, Juergens H, Fruehwald M, Groll AH. Delay in B-lymphocyte recovery and function following rituximab for EBV-associated lymphoproliferative disease early post-allogeneic hematopoietic SCT. Bone Marrow Transplant 2009; 43: 679-84.

9. Heslop HE, Slobod KS, Pule MA, et al. Long-term outcome of EBVspecific T-cell infusions to prevent or treat EBV-related lymphoproliferative disease in transplant recipients. Blood 2009; 115: 925-35.

10. Heslop HE. How I treat EBV lymphoproliferation. Blood 2009; 114: 4002-8.

11. Allen U, Barton M, Beyene J, et al. Gene expression using microarrays in transplant recipients at risk of EBV lymphoproliferation after organ transplantation: preliminary proof-of-concept. Pediatr Transplant 2009; 13: 990-8.

\section{Address for correspondence}

Lidia Gil MD, PhD

Department of Haematology

Poznan University of Medical Sciences

Szamarzewskiego 84

60-569 Poznań, Poland

tel. +48618549571

$\mathrm{fax}+48618549578$

e-mail: lidia.gil@skpp.edu.pl

Submitted: 26.02 .2012

Accepted: $\quad$ 7.05.2012 